\title{
Asociaciones de padres y seguimiento multidisciplinar de la gran prematuridad
}

\author{
Parents Associations and multidisciplinary \\ follow-up of very preterm infants
}

\section{Palabras clave \\ Gran prematuridad, bebés grandes prematuros, nacidos extremadamente pretérmino, asociaciones de padres de niños nacidos prematuros, seguimiento sociosanitario de la gran prematuridad.}

\section{Keywords}

Very preterm birth, very preterm infants, extremely preterm children, associations of parents of premature infants, follow-up of very preterm infants.
El texto que se presenta a continuación tiene su origen en una ponencia presentada en el "I Curso Multidisciplinar en la Atención al Seguimiento de niños nacidos con un peso < 500 g.", organizado por la Dra. Salas del Servicio de Neonatología del Hospital Universitario La Paz de Madrid ${ }^{\mathrm{I}}$ en marzo de 2012, donde se me invitó a participar como representante del movimiento asociativo de la prematuridad $^{2}$.

Este texto se hace eco de esa demanda por lo que a lo largo del mismo voy a hablar en primera persona y en

I. Un servicio que tiene una enorme importancia para el movimiento asociativo de la prematuridad en nuestro país. Porque fue la "incubadora" de dicho movimiento; el lugar donde un 23 de agosto de I999 un grupo de familias elaboramos los estatutos de la primera asociación que se creó en España y que, desde esos primeros momentos, contó con la colaboración de todo el equipo de Neonatología. Hoy, los/as excelentes profesionales que componen este Servicio, siguen apoyando decididamente la labor de las todas las asociaciones de padres que trabajamos en el ámbito de la prematuridad.

2. En concreto, como Vicepresidenta de la Asociación de Padres de Niños Prematuros (APREM). APREM, de ámbito estatal, es la primera asociación que se creó en España para defender los derechos de estos menores y de sus familias y, actualmente, sigue siendo la más numerosa. APREM colabora con las otras cinco entidades de padres de niños prematuros que existen en nuestro país: Apreand, Apremate, Avaprem, Pregran y Prematura. Quiero agradecer aquí el apoyo recibido de la Junta Directiva de APREM para la redacción de este documento.

\section{Concepción Gómez Esteban} <conchagomez@cps.ucm.es>

Presidenta de Alianza Aire y vicepresidenta de APREM Profesora Titular de la Universidad Complutense de Madrid
Para citar:

Gómez Esteban, C. (2OI4):

"Asociaciones de padres y seguimiento multidisciplinar de la gran prematuridad". Revista Española de Discapacidad, 2 (I): 203-2I3.

<http://dx.doi.org/I0.5569/23405IO4.O2.OI.I $2>$ 
nombre de las Asociaciones de Padres de Niños Prematuros para poner de manifiesto cuáles son las mejores prácticas en la atención que se presta a esos menores y para mostrar el papel que, a nuestro entender, pueden y deben jugar nuestras entidades en todas las cuestiones relacionadas con la mejora de la situación de los/as nacidos/as muy prematuramente y de sus familias.

Espero también que estas reflexiones sirvan para conocer mejor las consecuencias de la gran prematuridad y el nacimiento con bajo peso $^{3}$, un problema social emergente, sin duda, por la magnitud del número de nacidos ${ }^{4}$; la morbimortalidad, discapacidad, trastornos y dificultades socioeducativas que pueden presentar o desarrollar en un futuro estos neonatos; el coste socioeconómico que conlleva su adecuada atención; el impacto de todo ello en la vida de las familias; e incluso, los dilemas éticos que la gran prematuridad puede plantear a las ciencias de la salud y a la sociedad en general.

3. La Organización Mundial de la Salud (OMS) define como "recién nacido prematuro o pretérmino" al neonato cuyo parto se produce entre la semana 22 y la 36.6 de gestación y "nacido a término" cuando el parto se produce desde la semana 37 a la 42 .

La Sociedad Española de Neonatología (SENeo) y diversos autores incluyen dentro de la categoría de nacidos con $<$ I 500 g. a los siguientes colectivos: los muy pretérmino o muy prematuros, nacidos con menos de 32 semanas de edad gestacional y a los recién nacidos extremadamente pretérmino o prematuros extremos de $<28$ semanas de gestación. Si atendemos al peso al nacimiento, se incluye a los nacidos de muy bajo peso, bebés de entre I.000 y I.499 g. al nacimiento; los de extremo bajo peso, nacidos con < <.000 g.; y los nacidos con excesivo extremado bajo peso o "microprematuros" nacidos con $<750 \mathrm{~g}$. En adelante, cuando me refiera a los niños nacidos con < 500 g. lo haré de manera genérica, refiriéndome al conjunto de estos colectivos, aunque sus condiciones de salud puedan ser muy diferentes.

Hay que señalar que la aproximación más adecuada para conocer la madurez del neonato es combinar la edad gestacional con el peso al nacer, siendo los niños con bajo peso para la edad gestacional -nacidos por debajo del percentil ıo para la edad de gestación- los que suelen tener peor pronóstico. Pese a ello, hay que destacar que para todos los nacidos prematuramente o con bajo peso existe un gradiente de riesgo, incluso para los nacidos a término temprano -por debajo de la semana 38 de gestación-, como han puesto de manifiesto recientemente algunos estudios internacionales.

4. Según el Instituto Nacional de Estadística, de los nacidos en 20I2, un total de 4.08 I lo hicieron con <I 500 g. de peso; una cifra más reducida que la de años anteriores, dado que desde 2009 se viene produciendo una disminución de los partos prematuros en nuestro país.
Además, quisiera que las propuestas de las asociaciones de padres que se recogen a continuación contribuyeran a potenciar el desarrollo los nacidos con < 500 g. de peso y dar a conocer a los/as profesionales y a la opinión pública algunos aspectos de la complejidad y heterogeneidad de sus condiciones de salud. Pero antes de plantearlas, es necesario señalar una cuestión que vertebrará toda mi exposición: que como familias de niños nacidos prematuramente, queremos implicarnos activamente en el cuidado y en el seguimiento sociosanitario y educativo de nuestros hijos desde su nacimiento y su estancia en las Unidades de Neonatología y durante el tiempo que sus circunstancias lo requieran. No solo porque se trate de un derecho de estos menores sino porque somos conscientes del papel crucial que jugamos en el cuidado de su salud y de su calidad de vida a lo largo de todo el desarrollo madurativo. También porque consideramos que no cabe hablar de un abordaje multidimensional ni de un enfoque multidisciplinar de la prematuridad si no se cuenta con las familias y sus asociaciones y con su específico conocimiento experto.

\section{El proceso de seguimiento desde los cuidados centrados en la familia}

Las asociaciones de padres entendemos que la atención y el seguimiento ideal para nuestros hijos es aquel que proporciona al niño y a su entorno familiar todo lo que necesita, cuándo y cómo lo necesita. Y que, para lograrlo, el enfoque debe ser transversal: toda actuación debe ser diseñada e implementada desde la perspectiva de que el niño y su familia configuran una unidad y, por tanto, hay que valorar el impacto que cada actividad tiene para cada uno de los integrantes de dicha unidad.

En coherencia con este planteamiento general, consideramos que el propósito principal de un programa de seguimiento para los nacidos con $<$ I 500 g. es la atención integral coordinada 
al niño de alto riesgo biológico y a su familia con la finalidad de lograr la optimización de su estado de salud, el pleno desarrollo de sus potencialidades y su plena integración social. Se trata de observar el crecimiento y desarrollo de estos niños a lo largo de todo su proceso madurativo para prevenir y/o detectar precozmente las posibles alteraciones y trastornos e intervenir tempranamente y con la mayor efectividad y equidad; considerando al niño no a partir de sus posibles déficits sino de sus aspectos positivos, manteniendo en la medida de lo posible expectativas esperanzadoras sobre su desarrollo y contribuyendo al mayor bienestar de su familia. Un programa donde la familia tenga el derecho de tomar decisiones basadas en la mejor información y evidencia científica disponible y las asociaciones que representan a las familias puedan aportar su experiencia en cada etapa del proceso.

Por otra parte, entendemos que un programa de seguimiento que se realice bajo estas condiciones y esté coordinado no solo es positivo para el mejor desarrollo del bebé y para el bienestar de su familia, sino que evita repeticiones de pruebas y exploraciones, permite consensuar diagnósticos y prescripciones, $\mathrm{y}$, si incluye la recogida y análisis sistemático de datos, beneficia también a todas las entidades y profesionales implicados en este proceso al incrementar su conocimiento de las dificultades específicas que potencialmente pueden sufrir estos menores y contribuir a la mejora paulatina de los resultados del cuidado que se les presta.

\section{Propuestas de las asociaciones de padres para mejorar la atención neonatal y el seguimiento de los/as menores/as nacidos/as con $<1500 \mathrm{~g}$.}

A continuación presentaré las cuestiones que planteamos las familias de estos menores con el objetivo de potenciar su adecuado desarrollo e integración socio-familiar. No obstante, hay que señalar que las familias somos conscientes, por una parte, de las diferencias existentes entre los centros que componen la red asistencial que atiende a los nacidos con < 500 g. y de la dificultad de establecer muchas de nuestras propuestas en un contexto de restricción económica como el actual y en un país caracterizado por grandes diferencias entre comunidades autónomas a la hora de organizar la atención infanto-juvenil (lo que algunos expertos denominan "puntos negros" de esta atención y que generarían inequidades para los niños, sus familias y los profesionales que les atienden). Y, por otra, de las resistencias que, aunque cada vez tienen un carácter más minoritario, siguen existiendo en algunos medios a la hora de reconocer el papel protagonista que deben tener las familias en el cuidado del hijo o que se expresan en la dificultad para valorar adecuadamente las funciones que desarrollan las asociaciones que las representan. Ahora bien, ser consciente de estos problemas estructurales, y de algunos que esperemos que sean solo coyunturales, no implica renunciar a los derechos ni a mejorar la atención dirigida al colectivo de nacidos con <I 500 g. y a sus familias5.

\section{Buenas prácticas para mejorar la atención neonatal y el seguimiento de los nacidos con $<1500 \mathrm{~g}$.}

Aunque el objeto de este texto es analizar la atención y el seguimiento a realizar a estos bebés tras el alta de la Unidad de Neonatología, creo necesario decir unas palabras sobre el período previo, que se corresponde con el ingreso del bebé en la Unidad tras el nacimiento y la

\footnotetext{
5. Dejar constancia aquí de otro tema que nos preocupa a las asociaciones sobre el seguimiento sanitario a largo plazo de algunos menores, por la intolerable discriminación que supone para un colectivo tan potencialmente vulnerable como el de los nacidos con < 500 g. Según nos han transmitido algunas familias que tienen seguros sanitarios privados, hay compañías que no admiten como asociados a los hijos que nacen prematuramente, salvo que se pacte que nunca van a tener que tratarles de problemas relacionados con su condición neonatal.
} 
estancia de su familia en Neonatología. Cinco son las propuestas relativas a ese período que las asociaciones consideramos fundamentales para las familias. Se trata de propuestas que formarían parte de lo que entendemos debe ser la atención temprana a prestar al menor y a su entorno familiar. Pedimos:

- $\quad$ Planes de prevención de los partos prematuros y control y seguimiento de todos los embarazos de riesgo con independencia de las circunstancias maternas.

- Programas para familiarizar a los padres con las Unidades de Neonatología. Previo al ingreso de la Unidad, si hay tiempo para ello, o inmediatamente después, sería muy positivo para atenuar el impacto de un nacimiento pretérmino que el personal sanitario explicara qué es y cómo funciona ese espacio altamente tecnificado que son las Unidades de Neonatología y que contestara a algunas de las cuestiones que pueden generar inquietud a las familias, que se enfrentan a una experiencia tan poco conocida aún y tan traumática como un nacimiento en estas condiciones. Al menos, sería deseable que se entregara a los padres información sobre las Unidades y su funcionamiento adaptada a las peculiaridades de diferentes culturas y redactada en diferentes idiomas.

- Acompañamiento psicológico para la familia desde el momento en que se prevea un parto prematuro. El objetivo de este apoyo es evaluar lo antes posible las reacciones de estrés parental y la forma como se establece el vínculo con el hijo, así como potenciar los factores de resistencia y protección familiares. El acompañamiento se debe ofrecer como un recurso a disposición de los padres e incluir tanto ayuda individualizada como grupos de apoyo emocional que cuenten con la colaboración de padres "veteranos". Pensamos que este apoyo especializado tiene beneficios también para el personal de las Unidades que, sin duda, se ve abocado en algunas ocasiones a enfrentar situaciones anímicas que se desbordan.

Y en relación con esta demanda, añadir que queremos que otros profesionales y especialidades se integren plenamente en el trabajo y en la vida cotidiana de las Unidades de Neonatología. Desde el amplio sentido que concede la OMS a la condición de salud, consideramos que los centros sanitarios en general, y las Unidades de Neonatología en concreto, deberían ser entornos más 'permeables', donde se trabajase en equipo con otros profesionales que, como los del ámbito de la psiquiatría, la psicología, el trabajo social, la fisioterapia, la estimulación, etc., también proporcionan salud y cuyo trabajo resulta imprescindible en la evolución de los niños nacidos muy prematuramente.

- Implementación de un modelo de cuidados centrados en el desarrollo y en la familia. Consideramos que la estancia de las familias y de los neonatos en las Unidades de Neonatología se debe orientar en función del llamado "Modelo de Cuidados Centrados en el Desarrollo y en la Familia”, que incluye un conjunto de prácticas tales como unidades abiertas 24 horas a los padres, acceso de otros miembros de la familia, fomento de la lactancia materna y creación de bancos de leche donada, aplicación del método canguro y de la técnica "piel con piel”, cuidado de las condiciones ambientales de la Unidad, métodos para minimizar el dolor del neonato, espacios adecuados para el confort de las familias, posibilidad de hospitalizar a la persona que ejerza de cuidador/a con el hijo, etc. Aunque existen otros modelos de cuidados, pensamos que aún no hay evidencia científica clara sobre sus bondades, pero sí de su alto coste, y que no está claro el papel que se adjudica a la familia y a su bienestar en esos modelos.

En relación con el proceso cuyo análisis nos ha reunido aquí, el seguimiento multidisciplinar de los nacidos con < I 500 g., las asociaciones de 
padres quisiéramos que se estructurara tomando en consideración las siguientes propuestas:

\section{- Realización de Escuelas de Padres} intrahospitalarias: Aunque más adelante hablaré de sus funciones, señalar aquí la necesidad de que se realicen escuelas de padres donde puedan asistir las familias cuyos hijos vayan a recibir pronto el alta de la Unidad. Estas Escuelas se deben programar contando con la colaboración de representantes de las asociaciones de familias y de padres y madres veteranos para facilitar la comunicación y transmisión de la información y para crear redes de apoyo entre familias que se prolonguen más allá del alta hospitalaria del hijo.

- Alta precoz del bebé: Valoramos que desde las Unidades de Neonatología se fomente el alta precoz de estos niños siempre que (I) se cumplan las condiciones que lo permitan, (2) los padres se sientan plenamente seguros con esta situación y (3) puedan recibir apoyo sociosanitario en su domicilio. Pensamos que esta práctica, que facilita una más pronta integración del bebé en el entorno familiar, ayuda a normalizar la vida familiar y minimiza los períodos de separación familia-hijo que a menudo son doblemente traumáticos como sucede, por ejemplo en caso de partos múltiples, donde no es infrecuente tener un bebé todavía hospitalizado y otro/s ya en el hogar.

Además, el alta precoz tiene otros beneficios como la posibilidad de conciliar en mejores condiciones la vida familiar, laboral y personal, al permitir que los padres puedan recibir más fácilmente la ayuda que pudiera prestarles la red de parientes y amigos; incrementa la tasa de lactancia materna; disminuye la posibilidad de contraer infecciones nosocomiales; $y$, al suponer una reducción en los días de hospitalización, abarata el coste económico de la atención sanitaria neonatal que reciben estos bebés.

- Visitas al hospital coordinadas: Como el período inmediatamente posterior al alta de la Unidad exige muchas visitas al hospital para realizar el protocolo de seguimiento médico de los hijos nacidos con < 500 g., habría que garantizar la coordinación entre las especialidades que participan en este proceso para lograr que en un mismo día sea posible atender todas las citas médicas o a varias de ellas. No solo se trata de hacer menos complicada una crianza que suele ser difícil, sino de evitar, en lo posible, el riesgo de que niños tan vulnerables puedan contagiarse de enfermedades respiratorias agudas y otras dolencias que les afectan especialmente, al tener que permanecer en lugares donde hay personas que pudieran estar infectadas.

\section{- Coordinación interdisciplinar e} intersectorial de profesionales y entornos: En relación con el punto anterior, decir que si hay una demanda crucial para las asociaciones de padres es la necesidad de que exista una coordinación intra y extrahospitalaria entre los profesionales, especialidades y entornos que trabajan con nuestros hijos. Una coordinación que debería ser impulsada por los poderes públicos, estableciendo un modelo consensuado, estable y en gran medida estandarizado de funcionamiento, que incluya un control de calidad de los procesos y de sus resultados.

Entendemos que la coordinación no se debe plantear, como viene siendo habitual, como una actividad que vincula a algunos profesionales concretos que voluntariamente deciden colaborar en esta dirección, sino que debería ser intersectorial; esto es, impulsada por los responsables de las áreas organizativas de las que dependen las políticas sanitarias, de servicios sociales y educativas. Una labor que exige establecer conjuntamente la coherencia de dichas políticas, diseñar programas comunes y protocolos, racionalizar el uso de los recursos y medios y destinar los que sean necesarios para desarrollar adecuadamente todas las actividades propias del seguimiento. Para 
nosotros, el objetivo primordial es evitar los desfases y dilaciones en la atención a estos menores y posibilitar que puedan recibir lo más tempranamente posible la mejor y más adecuada atención en cada caso.

- Información a las familias sobre el proceso de seguimiento antes de recibir el alta de la Unidad: Una de las primeras medidas de un programa de seguimiento coordinado debería consistir en informar adecuadamente a las familias antes de que el hijo reciba el alta sobre los objetivos, servicios y especialidades implicados en el proceso de seguimiento sociosanitario y educativo de los menores nacidos con $<$ I $500 \mathrm{~g}$.

Esta es una de las principales funciones que atribuimos a las escuelas de padres porque, conocer a fondo en qué va a consistir este proceso, evitaría el "peregrinaje" 6 al que se han visto y se ven abocadas todavía muchas familias en nuestro país. Una falta de información y un "peregrinaje" que no solo implica una angustia y un estrés añadidos a la crianza de un hijo gran prematuro sino que, a veces, retrasa de forma muy considerable el acceso a los recursos y servicios destinados a la atención de estos niños.

- Designación de unla profesional de referencia para todo el proceso de seguimiento: La coordinación exige también que las familias cuenten con un/a profesional de referencia que pueda realizar para el equipo interdisciplinar intra e interhospitalario que atiende al niño y para los padres funciones similares a las atribuidas desde un enfoque biopsicosocial de cuidados al "gestor de caso", figura guía, nodo de comunicación y coordinación de

6. Este es el término coloquial con el que los padres se refieren a menudo a la búsqueda desorientada de información a la que se ven obligadas muchas familias por carecer de un asesoramiento adecuado antes de que el hijo sea dado de alta en la Unidad. Como en muchos hospitales no informan de la existencia de asociaciones de padres, las familias no suelen contar tampoco con este recurso de orientación. los diferentes entornos que participan en el cuidado que se presta a estos menores.

- Derecho de los padres a tomar decisiones informadas: Por otra parte, las familias consideramos que debemos disponer de información actualizada y lo más consensuada posible sobre el desarrollo de nuestros hijos, sus condiciones actuales, su posible evolución, las alternativas al tratamiento, etc. y a participar en el establecimiento de los objetivos y la evaluación de los programas y terapias que se les puedan aplicar. Queremos que se nos informe sobre nuestros derechos y que, en último extremo, se respete la libertad de los padres para tomar decisiones informadas sobre la salud de sus hijos? (lógicamente, siempre que estas no entren en colisión con el derecho del niño a la salud y al bienestar general).

- Incrementar la duración del proceso de seguimiento y adaptarlo a cada caso: $\mathrm{Si}$ como algunos expertos piensan la gran prematuridad es un hecho irreparable, por cuanto es una condición que potencialmente afecta a la salud global de un individuo y a todo su ciclo vital, entendemos que el seguimiento interdisciplinar de los nacidos con < 500 g. debería ir más allá de los 7-8 años en los que, en el mejor de los casos, se establece actualmente en nuestro país. Esta cuestión nos importa mucho porque las asociaciones de padres sabemos que hay trastornos que pueden aparecer o manifestarse según avanza el proceso de desarrollo; por ejemplo, al inicio de la pubertad y de la adolescencia, etapas en las que no hay previstos protocolos o programas de seguimiento estructurados, pese a que algunos de estos menores

7. Sería deseable que se respetaran las decisiones parentales especialmente cuando las familias optan por un tratamiento sobre el que no hay acuerdo entre los especialistas que atienden a sus hijos. A veces los padres se encuentran con la dificultad añadida de tener que ocultar algunos de los tratamientos pautados al hijo para evitar conflictos entre profesionales, con el problema que esta situación supone para el correcto seguimiento de la evolución del desarrollo del menor. 
necesitarían supervisión médica y, sobre todo, psicopedagógica (afortunadamente, algunos hospitales españoles ya han iniciado proyectos de este tipo, similares a los que se realizan en otros países desde hace décadas).

Las asociaciones somos plenamente conscientes de las repercusiones negativas que pueden tener los programas que contemplen revisiones periódicas para estos menores solo por el hecho de que hayan nacido con <I 500 g.; consecuencias tales como presentar la prematuridad como una marca indeleble, conllevar riesgo de estigmatización de todo el colectivo, funcionar como profecías autocumplidas, o implicar una mayor sobrecarga y ansiedad para las familias que necesitan olvidar tan traumáticos nacimientos, y que todo ello puede repercutir en el desarrollo del hijo y en la relación que se establece que con él. Pero, pese a todo ello, creemos que nuestros hijos se pueden beneficiar de un seguimiento sociosanitario y educativo más amplio, realizado siempre según su posible estado de salud y su evolución. También pensamos que estos programas pueden ser de utilidad para otros nacidos prematuramente porque, hoy por hoy, la mejor manera de conocer la complejidad intrínseca de lo que en rigor debemos considerar las "prematuridades" es obtener información minuciosa, exhaustiva y basada en criterios consensuados sobre el desarrollo y la evolución de estos menores. Entendemos que esta es una de las principales vías para identificar adecuadamente los riesgos y llegar en el futuro a una asistencia sociosanitaria personalizada que permita prevenir, realizar revisiones selectivas e individualizar el seguimiento y los apoyos, así como disminuir el coste de todas estas intervenciones.

- Facilitar la Atención Temprana que tanto beneficia a nuestros hijos y a nuestras familias: Por último, en esta relación de buenas prácticas que no pretende ser exhaustiva, señalaría un aspecto que nos preocupa sobremanera a las asociaciones: el posible retraso en acceder a los Servicios de Atención Temprana (AT) ${ }^{8}$ en el caso de menores, que, como los recién nacidos de alto riesgo biológico, tienen una mayor probabilidad de presentar durante su infancia desfase madurativo, dificultades sensoriales, de neurodesarrollo, etc. A diferencia de lo que consideran algunos neonatólogos, y mientras no haya evidencias científicas claras que lo desaconsejen para alguno de los grupos que componen tan heterogéneo colectivo, creemos que al menos todo bebé que nazca con $<32$ semanas de gestación o $<$ I 500 g. debe tener concertada antes de recibir el alta de la Unidad una valoración diagnóstica como parte del protocolo de seguimiento; una práctica de prevención de secuelas que está mucho más extendida en la red sanitaria pública que en la privada.

La valoración debe ser realizada por equipos multidisciplinares expertos en programas de rehabilitación y estimulación motora-sensorial precoz y en abordajes multidimensionales especializados en la detección y tratamiento de los problemas surgidos en el desarrollo de bebés en situación de riesgo ${ }^{9}$.

8. Algunos datos recientes publicados por las organizaciones que trabajan en este campo sugieren que la prevención secundaria está fallando porque todavía en muchas CCAA hay un elevado porcentaje de menores en situación de riesgo biológico que ingresan en los Servicios de Atención Temprana a una edad superior a los dos años, con el grave quebranto que este retraso supone para poder mejorar su salud (por supuesto, no siempre se trata de menores nacidos muy prematuramente). 9. Defendemos esta medida desde la responsabilidad que tenemos como representantes de los padres con hijos nacidos prematuramente y tras valorar tanto los inconvenientes como las ventajas que para los niños y para las familias puede suponer, no tanto que los hijos tengan que acudir a realizar esta valoración diagnóstica, sino que, en algunos casos, deban de asistir a revisiones periódicas y/o realizar un programa más o menos prolongado de estimulación o rehabilitación. (Entre otros datos que avalan nuestra propuesta, recordaría que el riesgo de retraso neurológico es posible incluso cuando las pruebas de control son normales; que el desarrollo psicomotor de los prematuros de muy bajo peso no permite descartar con certeza la presencia de secuelas neurológicas al menos hasta los dos años; o que todavía no hay pruebas concluyentes de que el riesgo de discapacidad haya disminuido claramente en nuestro país para los nacidos con < Io०० g.). 
Obviamente la atención posterior al menor dependerá de esa valoración y de la necesidad, en su caso, de ayudar a superar el desfase madurativo del bebé, supervisar la posible aparición de situaciones de riesgo social, prevenir trastornos, limitaciones funcionales y/o discapacidades y reducir la severidad de estas; en síntesis, actuaciones todas orientadas a intervenir tempranamente ${ }^{\text {Io }}$ para paliar la posibilidad de dependencia futura en esta población, con su alto coste económico y en calidad de vida.

Las asociaciones de padres defendemos que la AT se garantice como un servicio público, universal, gratuito e integral del que puedan beneficiarse todos los niños en situación de riesgo y sus familias. Concordamos con algunos expertos en que, desde la perspectiva de la trayectoria vital de la persona, la AT constituye el primer conjunto de servicios en los que se manifiesta la política social del país respecto a un ciudadano concreto; y pensamos que el retraso madurativo y las limitaciones en la actividad de un bebé se pueden convertirse en discapacidad como consecuencia de su interacción con un ambiente que no le proporciona el suficiente apoyo, como plantea la OMS. Por tanto, creemos que, en ningún caso, las políticas de austeridad y los enfoques económicos cortoplacistas pueden justificar recortes en este tipo de recursos, que son vitales para el buen desarrollo de nuestros hijos y también para el bienestar familiar.

Porque entre los beneficios que proporciona la AT está, además, el apoyo general a la familia. A menudo, los profesionales de estos servicios, al tener un contacto más frecuente con los padres, pueden establecer una mayor confianza y comunicación

Io. Como hemos dicho, desde nuestro punto de vista, la AT se debe iniciar desde el momento en que se suscita la posibilidad de un parto pretérmino o bien, una vez producido este, en las Unidades de Neonatología cuando se trata de colectivos de alto riesgo o con deficiencias constatadas. En algunos hospitales ya existen programas de rehabilitación y estimulación motora-sensorial precoz, que se inician mientras el bebé se encuentra hospitalizado en la Unidad y no terminan hasta que es derivado después del alta a equipos y/o centros más específicos. con ellos y realizar una labor de apoyo al conjunto familiar. No solo los padres están más tranquilos respecto del desarrollo de sus hijos cuando disponen de este recurso especializado, sino que tienen más facilidad para exponer a estos profesionales los problemas que aprecian en el niño o surgen en la vida familiar (psicológicos, económicos, de convivencia, etc.). Los Servicios de Atención Temprana, por tanto, ayudan a las familias a afrontar este tipo de situaciones y a conocer los recursos disponibles para ello, contribuyen a reforzar las capacidades de los padres como cuidadores y a mejorar su calidad de vida y, en síntesis, a ofrecer al niño un entorno y una crianza mucho más saludables ${ }^{\mathrm{II}}$.

4. Las asociaciones de padres: un recurso comunitario esencial

Uno de los objetivos del "I Curso

Multidisciplinar en la Atención al Seguimiento de niños nacidos con un peso < I 500 g." que propició este documento, era dar a conocer la cartera de servicios y recursos para los niños de alto riesgo. En línea con ese objetivo, quiero señalar el importante papel que las organizaciones del tercer sector, y en concreto las de padres de nacidos prematuros, pueden jugar al constituir un recurso comunitario fundamental que permite que el apoyo y el

II. Para sensibilizar ante estos problemas e impulsar una mayor implicación de los poderes públicos, las asociaciones de padres, a iniciativa de APREM, elaboraron con ocasión del II Día Mundial de Concienciación sobre la Prematuridad ( 77 de noviembre de 20I0) el documento titulado "Manifiesto de las Asociaciones de Padres de Niños Prematuros de España”, solicitando a los Gobiernos central y autonómicos la elaboración y puesta en marcha inmediatas de un Plan de prevención y atención, integral y adaptado a las necesidades específicas de los menores de o a 6 años en situación de riesgo biológico, familiar o ambiental que garantizara a nivel estatal la igualdad real de oportunidades entre los niños que necesitan de esta atención. (Este Manifiesto, que constituye el primer documento consensuado por todas las asociaciones de padres de nacidos prematuros de nuestro país, ha sido firmado también por más de cien profesionales relacionados con la atención a los niños en situación de riesgo). 
cuidado que se brinda en las Unidades de Neonatología se prolongue más allá del alta hospitalaria.

Como es bien conocido, la prematuridad supone para muchas familias una carrera de obstáculos y de resistencia que no acaba cuando el hijo sale del hospital. Poder encarar estas dificultades, que a menudo se prolongan en el tiempo, con el apoyo de otros que ya las han vivido y que tienen la información y los recursos necesarios para afrontarlas, es un factor esencial de protección en el desarrollo del niño y en la normalización de la situación familiar. Por tanto, quiero animar desde aquí a los profesionales a que faciliten a sus pacientes el apoyo específico, no equivalente a ningún otro, que suponen las redes constituidas por las asociaciones de padres.

Asociaciones de padres que podemos realizar las siguientes funciones en relación con los cuidados neonatales y el proceso de seguimiento sociosanitario y educativo de los nacidos prematuramente:

- Proporcionar información contrastada sobre la prevención de los partos prematuros y sobre cualquier otra cuestión que los servicios de neonatología y pediatría o los profesionales del ámbito de los servicios sociales y educativos consideren de interés transmitir a las familias.

- Asesorar el diseño de espacios destinados a los padres con hijos ingresados en neonatología o cualquier otro tipo de instalaciones dirigidas a estos menores y a sus familias.

- Proporcionar atención a los padres cuyos hijos están ingresados en las Unidades de Neonatología, tanto a nivel individual como realizando grupos de apoyo emocional en colaboración con especialistas en esta materia. Continuar asistiendo a las familias una vez que el hijo recibe el alta y regresan con él al hogar.

- Asesorar a las Unidades de Seguimiento de los Servicios de Neonatología, a los Centros de Atención Primaria, los Centros Base, los Centros de Desarrollo Infantil y Atención Temprana, los Equipos de Atención Temprana y de Orientación Educativa y Psicopedagógica, entre otros, en aquellas cuestiones donde se requiera conocer el punto de vista de las familias.

- Sensibilizar a la opinión pública sobre las consecuencias de la prematuridad, los avances producidos en este terreno y otras cuestiones relacionadas, organizando junto con los profesionales actividades científicas o de otro tipo orientadas específicamente a las familias y al público en general como la celebración del Día Mundial de la Prematuridad.

- Realizar escuelas de padres en coordinación con el personal sociosanitario.

Las escuelas de padres son un importante recurso para las familias en el que las asociaciones podemos colaborar de manera muy destacada, aunque solo sea porque conocemos bien las circunstancias y la ansiedad que supone para los padres el regreso al hogar con un bebé nacido en estas condiciones y que ha contado para su cuidado, hasta ese momento, con importantes recursos humanos y técnicos ${ }^{\mathrm{I} 2}$.

En las escuelas de padres intrahospitalarias ya establecidas, las asociaciones impartimos charlas en las que, desde un enfoque positivo y preventivo y basándonos en la credibilidad que nos conceden las familias al conocer nuestra condición de padres "veteranos" - por tanto, de personas que hemos enfrentado circunstancias y retos muy similares a las suyos-, informamos a los asistentes sobre prácticas y medidas concretas que les ayuden a evitar el "peregrinaje" y a que

I2. Si bien la primera preocupación de las familias cuando se produce el alta hospitalaria es "el estado general de salud del hijo y su posible evolución futura", la segunda es "el miedo a no saber cuidar adecuadamente al hijo" y la tercera la "falta de información sobre cuestiones que luego las familias han visto que eran importantes para el desarrollo del niño, según los resultados de una encuesta promovida por APREM en 20I 0 cuyos resultados coinciden en gran medida con los de otros estudios realizados sobre el colectivo de los menores nacidos con <I $500 \mathrm{~g}$. 
la reorganización y adaptación familiar se realice de la manera más exitosa posible. Intentamos también reforzar el sentido de competencia y confianza de los padres para que afronten con el mejor estado anímico la adaptación al hogar y desarrollen lo más adecuadamente posible las funciones parentales específicas que necesitan los bebés nacidos muy prematuramente. Pero, sobre todo, destacaría el hecho de que estas charlas se constituyen en un espacio donde las familias con hijos ingresados en las Unidades de Neonatología pueden encontrarse emocionalmente con otros padres de niños prematuros, expresar más fácilmente sus temores y esperanzas y establecer redes personales y sociales de apoyo con los beneficios presentes y futuros que este tipo de contacto tiene para toda la familia.

Pero las asociaciones de padres podemos y queremos colaborar con los profesionales que atienden a nuestros hijos en muchos otros proyectos. A continuación relaciono algunas de las actividades que, como las anteriormente señaladas, ya se están desarrollado conjuntamente con distintos equipos sociosanitarios y educativos, e incluyo otros posibles ámbitos de actuación:

- Sensibilizar a la opinión pública y visibilizar los problemas asociados a la prematuridad; en su caso, actuar como un grupo de presión en apoyo de determinadas medidas o programas que redunden en la mejora de la atención que se proporciona a estos menores y a sus familias.

- Recomendar fuentes bibliográficas contrastadas y recursos audiovisuales a las familias, que cada vez requieren más información de este tipo y están mejor formadas.

- Difundir a través de canales como nuestras webs, grupos en Facebook o Twitter, eventos o información de interés enviados por entidades institucionales, grupos profesionales, equipos científicos, etc.

- Promover y participar en reuniones, jornadas, mesas redondas y en todos aquellos foros donde se dé a conocer la opinión de las familias y/o se solicite la transferencia de nuestros conocimientos.

- Funcionar como un nodo de comunicación y promover el trabajo en red entre los profesionales interesados en conocer la labor de las asociaciones de padres y/o colaborar con ellas en cuestiones relacionadas con la prematuridad.

- Impulsar y realizar estudios e investigaciones sobre prematuridad, mostrando nuestro apoyo a la presentación de proyectos científicos y sociales, solicitando la participación de nuestros socios en ellos o, en su caso, promoviéndolos directamente ${ }^{\mathrm{I} 3}$.

Aunque en nuestro país todavía las asociaciones que trabajamos desde el voluntario con estos menores y sus familias tenemos un tamaño reducido y estamos poco vertebradas, como se observa, es mucho lo que hacemos y más lo que podemos hacer si contamos con el apoyo y colaboramos de los profesionales que trabajan en el ámbito de la prematuridad. Y cuando hablo de "colaboración", la entiendo en un sentido amplio; principalmente, como el interés por propiciar una comunicación positiva, basada en el respeto, la confianza y el reconocimiento mutuo, que potencia la labor de ambas partes, arroja réditos para todos ${ }^{\mathrm{I}}$, $\mathrm{y}$ tiene como fin

I3. Tal es el caso del proyecto "Dificultades biosociales de la gran prematuridad y el bajo peso: Los/as menores de I 8 años nacidos/as con $\leq$ I. 500 g. en España" (ref. CSO2OI I24294), financiado por el Plan Nacional de I+D+i, que cuenta con la SENeo, el CERMI y APREM como entes promotores observadores. Con este proyecto esperamos aportar datos que contribuyan a conocer mejor el desarrollo de los grandes prematuros y, por tanto, que sean de interés para el proceso de seguimiento multidisciplinar de la salud de estos menores. I4. Contrariamente, como ya se dijo, nuestra experiencia es que todavía la colaboración con el paciente y con su entorno, y en especial con sus asociaciones, despierta resistencias. Detectamos dobles discursos y formas de "apoyar" a los padres que evidencian la actitud paternalista con la que aún algunos profesionales enfocan estas relaciones.

Hace falta que el afán de excelencia que guía las actuaciones en pro de la mejora de la supervivencia y el estado de salud de los recién nacidos muy prematuramente se dirija también a la mejora de las complejas y contradictorias relaciones que en algunos casos se establecen entre familias y personal sanitario; en términos generales, entre los sistemas expertos y los 
último la salud del menor y la calidad de vida de toda la familia.

Las familias son expertas en el cuidado de sus hijos, y las asociaciones que defendemos sus derechos y las apoyamos tenemos una visión y un tipo de conocimiento específico que no se puede elidir si se busca una atención multidisciplinar y de calidad para los menores nacidos con <I 500 g. Por eso, pensamos que no son solo los padres los que tienen que establecer un vínculo positivo con el hijo recién nacido, también tenemos que establecerlo las asociaciones y los profesionales, porque, como muestran algunos estudios hechos en España sobre las familias con niños en situación de riesgo, el aspecto fundamental para alcanzar la calidad de vida es la manera en que se prestan los servicios; o lo que es lo mismo, la forma en la que se establece la relación y la comunicación entre profesionales y familia. 